Setiawan, Y., Roozegar, M., Zou, T., Morozov, A. and Angeles, J., 2018, "A topology-change model of multi-speed transmissions in electric vehicles during gear-shifting," Mechatronics, Vol.55, pp.151-161 CC-BY-NC-ND; DOI 10.1016/ j.mechatronics.2018.09.004

\title{
A Topology-change Model of Multi-speed Transmissions in Electric Vehicles during Gear-shifting
}

\author{
Yuhanes Dedy Setiawan Liauw*, Mehdi Roozegar, Ting Zou, Alexei Morozov, Jorge Angeles \\ Centre for Intelligent Machines (CIM) and Department of Mechanical Engineering, \\ McGill University, 845 Sherbrooke Street West, Montreal, QC, H3A 0G4, Canada
}

\begin{abstract}
Recent developments in electric vehicles incorporate multi-speed transmissions to improve the electric vehicle range and performance. Similar to transmissions for fossil-fuel vehicles, those for their electric counterparts undergo topology changes during gear-shifting. However, this important phenomenon has been overlooked in the development of transmission mathematical models, which results in inaccurate prediction of the transmission dynamic response. In this study, a topology-change model based on the impulse-momentum relation is developed to address the topology change of multi-speed transmissions in electric vehicles during gear-shifting. An orthogonal complement is introduced to eliminate the non-working constraint forces. The velocity jump brought about by topology changes is given due attention. A case study is included, whereby the model is applied to a novel modular multi-speed transmission for electric vehicles. First, tests are conducted on the transmission experimental testbed; then, the test results are reproduced in simulation using the model developed herein. Moreover, the velocity jump of the gear-shifting is computed by means of the model. Subsequently, simulation of the transmission gear-shifting incorporating the velocity jump is conducted. It is shown that the topology-change model improves the accuracy of transmission mathematical models in predicting transmission dynamic responses during gear-shifting.
\end{abstract}

Keywords: Topology-change model; Electric vehicles; Multi-speed transmissions; Dynamic response; Gear-shifting

\section{Introduction}

Commercialized by the end of the 19th century, electric vehicles (EVs) were not popular until recently, due to rapid depletion of fossil fuel and new legislation for exhaust emissions [1 3]. Consequently, EVs have been developed to improve efficiency [4-10]. Electric medium- and heavy-duty vehicles (EMHVs), such as trucks and buses, are the focus of this paper. Recent advances in medium- and heavy-duty vehicles lie in electrification and hybridization because of high energy consumption and gas emission in these types of vehicles. In addition, an application of multi-speed transmissions (MSTs) has been of interest because EMHVs otherwise need large electric motors (EMs) to carry heavy loads. Improvement of EV range and performance due to the application of MSTs has been verified [11 16]. To illustrate the improvement, an MST developed by Antonov Automotive Technologies Ltd. in Warwick, U.K. was able to maintain the EM at efficiency values higher than $90 \%$. It was shown, as well, that the EV efficiency under the New European Driving Cycle increased by 15\% [14]. In addition, the contribution of MST application in EVs was validated experimentally with EVs in field tests [17, 18].

Multi-speed transmissions have a significant influence on the vehicle performance and efficiency. The development of MSTs has improved largely due to the advancement of computer simulation. Transmission mathematical models play an important role in simulation tests intended to predict the transmission dynamic response to various inputs and conditions. Simulation results largely depend on the accuracy of the transmission models; many of these are available [19 23]. However, one important phenomenon has been overlooked

\footnotetext{
* Corresponding author

Email address: yuhanes.liauw@mail.mcgill.ca (Yuhanes Dedy Setiawan Liauw)
} 
when modeling the transmission system, which results in unreliable predictions of the dynamic response, namely, the transmission topology changes during gear-shifting. Multi-speed transmissions are mechanisms with variable topology because the gear pairs under meshing are shifted during operation. Transmissiontopology changes occur when gears are shifted from one speed ratio to another. Changing the transmission topology from one to another gear ratio causes the system to undergo discontinuities; the dynamic behavior follows the current gear ratio. Modeling this type of system is challenging because different models and constraints need to be imposed for different topologies. This effect has been taken into account by means of a combination of different models for predicting the dynamic response of the system 24 27]. Nevertheless, the topology changes in multi-speed transmissions during gear-shifting are yet to be modeled, as this matter has not been considered in the development of transmission mathematical models.

Topology changes occur frequently in mechanical systems [28]. Failing to take this phenomenon into account in the system mathematical model leads to incorrect dynamic-behavior prediction because the generalized velocity undergoes jumps upon topology changes [26, 29|. For example, Mukherjee and Anderson tested a quadruple pendulum that undergoes a topology change [29]. The pendulum swung from rest and the last body was instantaneously grounded at a given instant. The simulation results show that the topology change brings about velocity jumps. In a similar way, another quadruple pendulum was tested with one of the joints locked at a certain instant [26]. Joint-locking leads to a topology change, which results in velocity jumps.

Two main topology-change models are available in multibody systems. The first is based on contact/impact mechanics, where spring-damping systems are used to represent the contact/impact area 30, 31]. The influence of topology changes can be understood by means of this model. Furthermore, the full history of contact/impact forces during topology changes can be obtained for thorough dynamic analyses. However, real-time simulation, which is needed to simulate the topology change with high fidelity in a natural time frame, cannot be achieved with this model due to its high computational cost. On the contrary, the second model lends itself to real-time simulation because it allows for larger time steps [32, 33]. This model is based on the impulse-momentum relation and the coefficient of restitution to obtain a discontinuous response upon topology changes. Nevertheless, this model still entails numerical problems in coping with discontinuous jumps in the system state [26].

A new and simple model to compute discontinuous jumps in system velocity during topology changes was proposed recently [26]. The model utilizes the impulse-momentum relation and the Lagrangian equations of the first kind, which leads to a system of differential-algebraic equations. In our study, a new topology-change model is developed to address the topology changes in EV MSTs during gear-shifting. The model derived by Guo and Wang [26] is adopted here, except that the impulse of the applied forces is not assumed to vanish because these forces in EV MSTs are the clutch torques, which are impulsive. Furthermore, an orthogonal complement is introduced to eliminate the non-working constraint forces. A case study is included, whereby the model is applied to a novel modular MST in EVs. In order to calculate the impulse, experimental and simulation tests are conducted by means of the transmission testbed and MATLAB/Simulink, respectively. A gear-shifting operation is recorded experimentally and replicated by means of a mathematical model in simulation. Moreover, the velocity jump of the gear-shifting is computed by means of the model. Subsequently, simulation of the transmission gear-shifting incorporating the velocity jump is conducted. The topology-change model improves the ability of transmission mathematical models to predict the transmission dynamic response during gear-shifting.

\section{Topology-change Model}

The model that allows the calculation of the velocity jump in EV MSTs due to topology changes upon gear-shifting is derived in this section. The system of interest in this study is a transmission system with linear constraints. The dynamics equations are integrated over the duration of a topology-change event to obtain the impulse-momentum relation. For a holonomic multibody system with $n$ generalized coordinates, arrayed in vector $\mathbf{q}$, and $s$ constraint equations, the position-level constraint equations are

$$
\phi(\mathbf{q})=\mathbf{0}_{s} \in \mathbb{R}^{s}, \quad \mathbf{q} \in \mathbb{R}^{n}, \quad n>s
$$

The velocity-level constraint equations can be obtained by differentiating Eq. (1) with respect to time:

$$
\frac{d \phi}{d t}(\mathbf{v})=\boldsymbol{\Phi} \mathbf{v}=\mathbf{0}_{s} \in \mathbb{R}^{s}
$$


where $\mathbf{v}$ is the array of generalized velocities, $\mathbf{v} \equiv \dot{\mathbf{q}}$, and $\boldsymbol{\Phi}$ the $s \times n$ Jacobian associated with the constraints at the position level, $\mathbf{\Phi}=\partial \phi / \partial \mathbf{q} \epsilon \mathbb{R}^{s \times n}$.

Moreover, the mathematical model of the constrained system can be expressed as

$$
\mathbf{M} \dot{\mathbf{v}}+\boldsymbol{\Phi}^{T} \boldsymbol{\lambda}=\mathbf{f}(\mathbf{q}, \mathbf{v}, t)
$$

with $\mathbf{M}$ denoting the $n \times n$ constant mass matrix, $\mathbf{f}(\mathbf{q}, \mathbf{v}, t)$ the array of applied forces, and $\boldsymbol{\lambda}$ the $s$-dimensional array of Lagrange multipliers. The Coriolis and centrifugal forces vanish because the Lagrangian equations and constraints are all linear.

Let $\mathbf{T}$ be an orthogonal complement [34] of $\mathbf{\Phi}$, and hence, $\mathbf{T} \in \mathbb{R}^{n \times n^{\prime}}$ with $n^{\prime} \equiv n-s$; therefore, $\mathbf{\Phi T}=\mathbf{O} \epsilon \mathbb{R}^{s \times n^{\prime}}$. Pre-multiplying both sides of Eq. (3) by $\mathbf{T}^{T}$, we eliminate the term in the Lagrange multipliers, thereby obtaining

$$
\mathbf{T}^{T} \mathbf{M} \dot{\mathbf{v}}=\mathbf{T}^{T} \mathbf{f}(\mathbf{q}, \mathbf{v}, t)
$$

Moreover, let

$$
\mathbf{v}=\mathbf{T w}, \quad \mathbf{w} \epsilon \mathbb{R}^{n^{\prime}}
$$

where $\mathbf{w}$ denotes the vector of independent generalized velocities, $\mathbf{w}=\dot{\mathbf{x}}, \mathbf{x} \in \mathbb{R}^{n^{\prime}}$ being the vector of independent generalized coordinates.

Substituting Eq. (5) into Eq. (4) leads to

$$
\mathbf{T}^{T} \mathbf{M}(\mathbf{T} \dot{\mathbf{w}}+\dot{\mathbf{T}} \mathbf{w})=\boldsymbol{\tau}(\mathbf{x}, \mathbf{w}, t) \epsilon \mathbb{R}^{n^{\prime}}
$$

with $\boldsymbol{\tau} \equiv \mathbf{T}^{T} \mathbf{f}$, or

$$
\mathbf{T}^{T} \mathbf{M T} \dot{\mathbf{w}}+\mathbf{T}^{T} \mathbf{M} \dot{\mathbf{T}} \mathbf{w}=\boldsymbol{\tau}(\mathbf{x}, \mathbf{w}, t)
$$

thus obtaining a model free of constraint forces.

Introduction of $\mathbf{w}$ results in a new mass matrix $\mathbf{I}=\mathbf{T}^{T} \mathbf{M T} \epsilon \mathbb{R}^{n^{\prime} \times n^{\prime}}$ in Eq. (7). In addition, the second term of the left hand side in Eq. (7) vanishes because $\mathbf{T}$ is constant. Equation (7) can then be expressed as

$$
\mathbf{I} \dot{\mathbf{w}}=\boldsymbol{\tau}(\mathbf{x}, \mathbf{w}, t)
$$

The generalized coordinates remain unchanged during a topology change, whereas the generalized velocities undergo a jump. Let $t^{-}$and $t^{+}$represent the instant just before and just after the topology-change event, henceforth referred to as simply the event. Furthermore, let the event occur at $t_{0}$, and the independent generalized coordinates at $t_{0}$ be $\mathbf{x}_{0}=\mathbf{x}\left(t_{0}\right)$. Integrating Eq. (8) from $t^{-}$to $t^{+}$to analyze the dynamics of the system during this period, we have

$$
\int_{t^{-}}^{t^{+}} \mathbf{I} \dot{\mathbf{w}} d t=\int_{t^{-}}^{t^{+}} \boldsymbol{\tau}\left(\mathbf{x}_{0}, \mathbf{w}, t_{0}\right) d t
$$

Let matrix $\mathbf{I}=\mathbf{I}_{0}$, which is constant in $\left[t^{-}, t^{+}\right]$; therefore, the integral of the first term in Eq. (9) is $\mathbf{I}_{0} \Delta \mathbf{w}$, $\Delta \mathbf{w}$ representing the velocity jump. We further introduce $\iota_{0}$ as the impulse of the applied force, i.e., the RHS of Eq. (9), which leads to

$$
\mathbf{I}_{0} \Delta \mathbf{w}=\iota_{0}
$$

The velocity jump $\Delta \mathbf{w}$ can now be readily obtained as

$$
\Delta \mathbf{w}=\mathbf{I}_{0}^{-1} \iota_{0}
$$




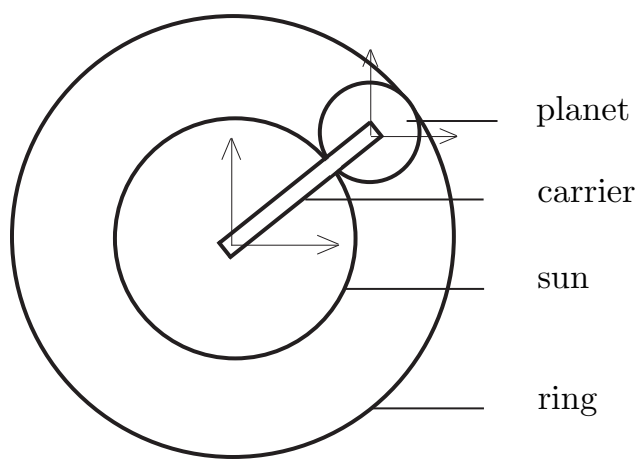

(a)

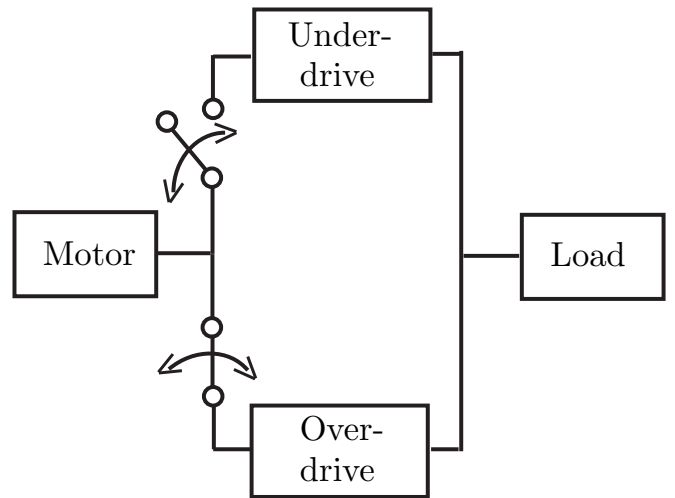

(b)

Figure 1: (a) A simple PGS; (b) a diagram of the MST

In order to find the velocity jump $\Delta \mathbf{w}$ in Eq. (11), the impulse $\iota_{0}$ needs to be first determined. As detailed above, $\iota_{0}$ is the time integral of $\boldsymbol{\tau}$, where $\boldsymbol{\tau}$ is $\mathbf{T}^{T} \mathbf{f}$ and $\mathbf{T}$ in Eq. (11) is an orthogonal complement of $\boldsymbol{\Phi}$ at the new topology. Both the applied forces $\mathbf{f}$ and $\mathbf{T}$ depend on the transmission design. In addition, the applied force $\mathbf{f}$ of the transmission depends on the input torque, the load torque and the clutch torques. The force $\mathbf{f}$ can be obtained in simulation by means of the transmission mathematical model. Experiments should be conducted to validate the results. Furthermore, the procedure can be reversed, namely, analyze a gear-shifting operation in the experimental testbed or in the transmission of interest, and replicate the gear-shifting in simulation by means of a mathematical model. The applied force $\mathbf{f}$ needed for gear-shifting as well as the impulse $\iota_{0}$ can then be obtained from the model. Moreover, the model in Eq. (11) is applicable to all types of gear-shifting operations, as the model does not limit itself to a specific transmission layout. It is apparent that the model depends on the constraints and the applied forces during gear-shifting. Therefore, once this information is available, regardless of the gear-shifting type, the velocity jump can be calculated.

A case study is included below to illustrate the foregoing approach. A gear-shifting operation in our testbed is recorded experimentally. The operation is then replicated in simulation by means of the mathematical model to find the applied force $\mathbf{f}$ needed for gear-shifting. Subsequently, the velocity jump $\Delta \mathbf{w}$ is computed.

\section{Case Study}

In order to illustrate the application of our topology-change model, a case study is included, whereby the model of Eq. (11) is applied to a novel modular MST intended for EVs [11]. The MST uses a planetary gear set (PGS). A simple PGS consists of four parts: a sun gear; a planet-carrier; a planet gear; and a ring gear, as shown in Fig. 1(a). The MST comprises two planetary gear trains, underdrive and overdrive, that are connected by the carrier 35]. An input motor and a load are used, as depicted in Fig. 1(b).

The MST is unique in its modularity. The number of speed ratios can be adjusted according to the vehicle need. Different numbers of PGSs can be installed in the underdrive and the overdrive gear trains. The MST considered in this paper is composed of two PGSs in parallel in both the underdrive and the overdrive gear trains, as shown in Fig. 2. The first and the second underdrive sun gears sit on a common shaft, those in the overdrive on another common shaft. The four PGSs are connected by a common carrier. Three planet gears are used in each PGS. A graph representation of the transmission, depicted in Fig. 3, illustrates the system topology when all clutches are open. The MST has four revolute pairs, 11 inertia elements, eight gear pairs (meshings), four ring clutches, and two multi-disk clutches. The inertia elements are numbered from 1 to 11. The gear pairs are depicted by dashed lines, whereas the revolute pairs by solid edges. Nodes and edges are used to account for the inertia elements and the kinematic joints, respectively. To give an illustration of a transmission topology change, the functional and graph representation for the first operation mode is given in Fig. 4. In addition, the gear ratios are changed by a combination of grounding the ring gears and connecting the carrier to the sun shafts. The former is done by a ring clutch, the latter by a multi-disk clutch. 


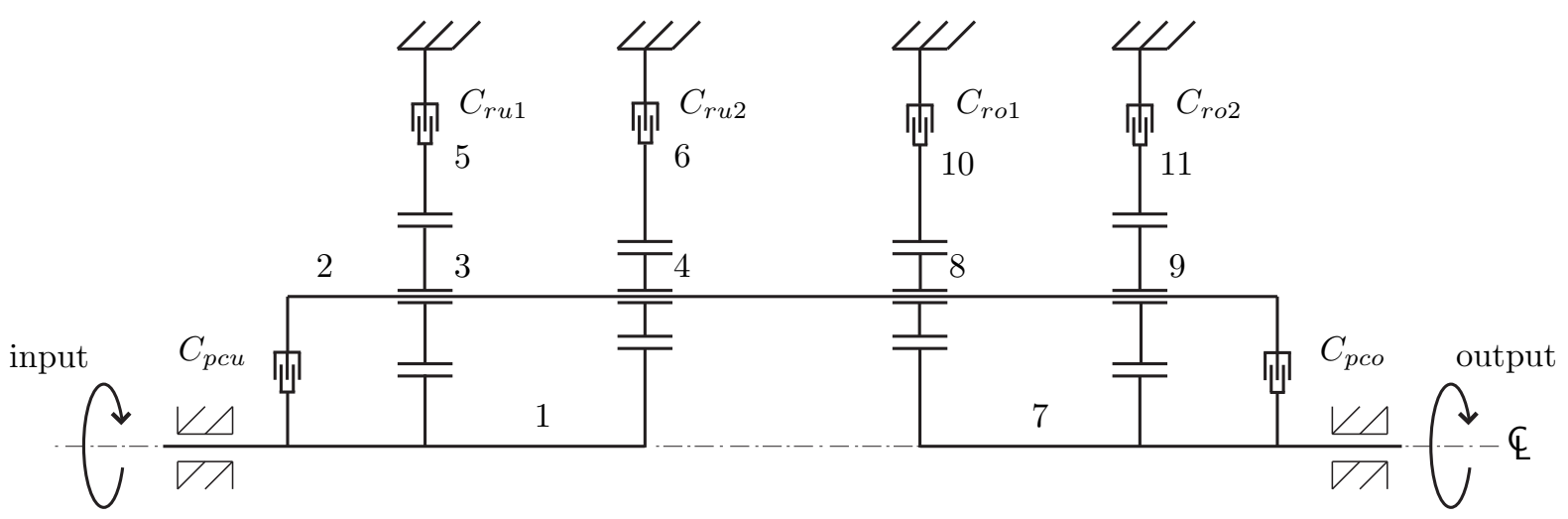

Figure 2: Functional representation of the transmission [23]

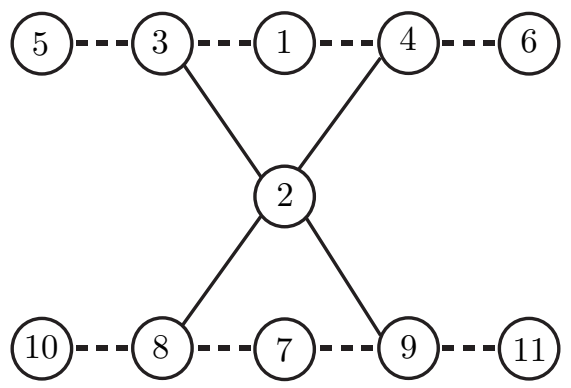

Figure 3: Graph representation of the transmission [23]

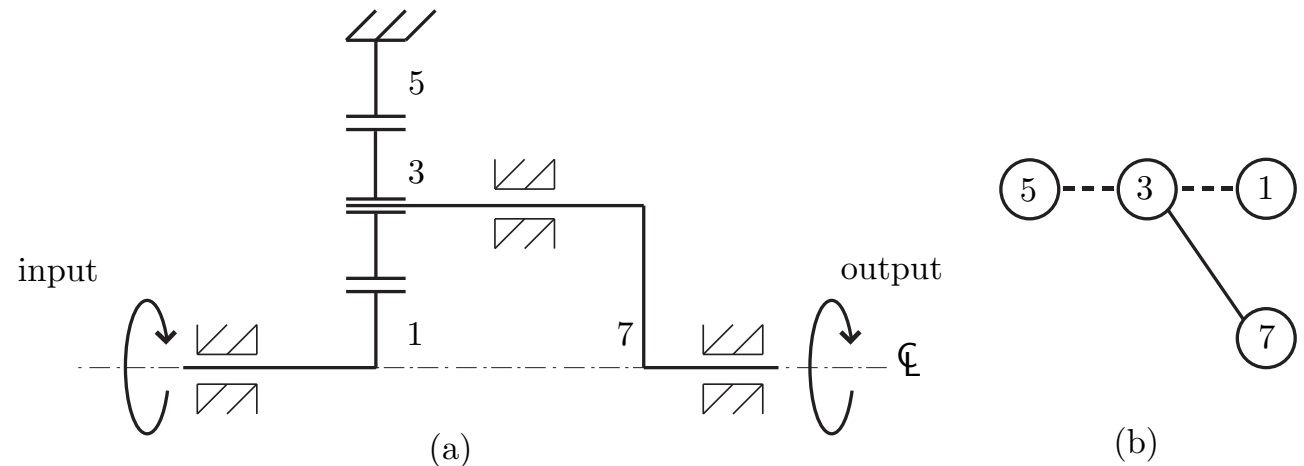

(a)

(b)

Figure 4: The first operation mode: (a) functional and (b) graph representation 23]

\subsection{Gear-shifting Mechanism}

To reduce the complexity of the problem and focus on the application of the topology-change model, gear-shifting in the underdrive gear train of the transmission shown in Fig. 15 is studied. The input comes from the common shaft on which the sun gears sit. In addition, the output torque is delivered by the common carrier, which is connected directly to the output shaft. Moreover, gear-shifting happens through switching of the ring clutches $C_{r u 1}$ and $C_{r u 2}$. The clutch state configuration is provided in Table 1. The underdrive gear train has two topologies. The system has the first topology when $C_{r u 1}$ is closed and the transmission operates with a 4:1 gear ratio. In turn, the system is in its second topology when $C_{r u 2}$ is closed and the transmission functions with a 2.67:1 gear ratio. Furthermore, the system topology transitions from one gear ratio to another when the on-coming clutch is engaging, while the out-going clutch is disengaging. In this study, the gear-shifting from the first topology to the second is of interest.

The transmission velocities jump during gear-shifting due to topology changes. For instance, ring gear 1, the ring gear in the first PGS, suddenly moves once $C_{r u 1}$ is disengaged because the planet gears are revolving around the internal meshing of the ring gear. In an ideal scenario, the second PGS is set ready when 


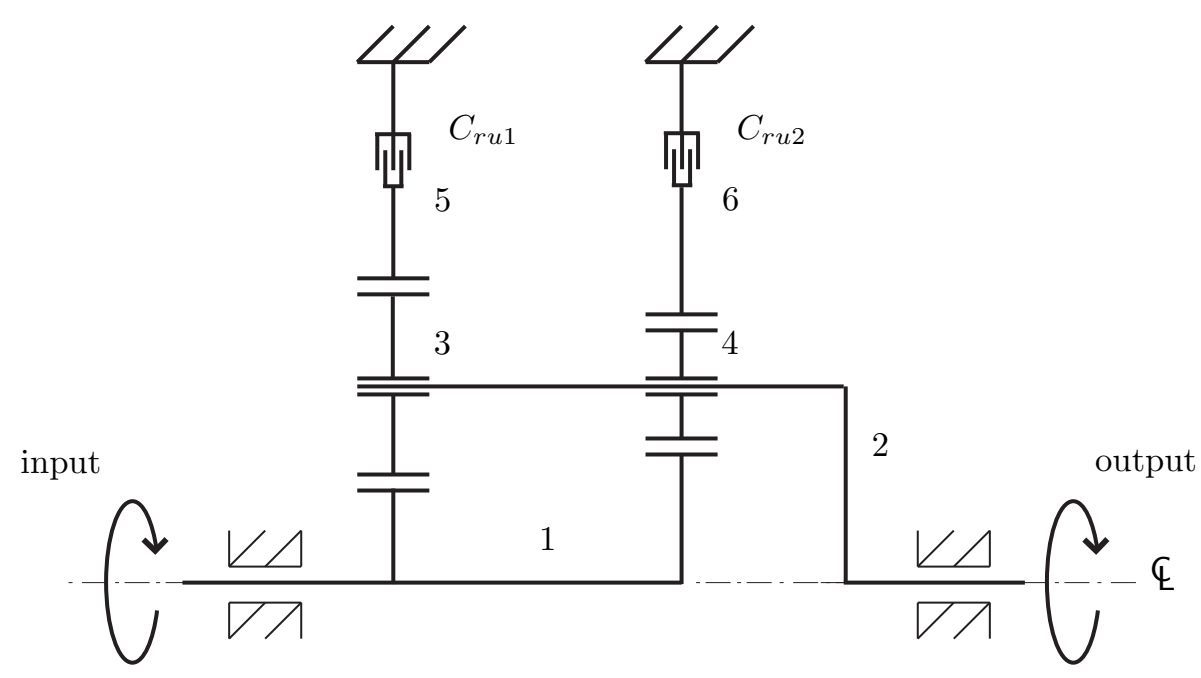

Figure 5: Functional representation of the underdrive gear train

Table 1: Clutch state configuration $(\times$ means closed $)$

\begin{tabular}{|c|c|c|c|}
\hline No & Gear ratio & $C_{r u 1}$ & $C_{r u 2}$ \\
\hline 1 & $4: 1$ & $\times$ & \\
\hline 2 & $2.6: 1$ & & $\times$ \\
\hline
\end{tabular}

ring gear 1 is released completely, namely, when ring gear 2, the ring gear in the second PGS, is grounded completely such that the transmission can operate with the second gear ratio. The optimum gear-shifting aims to disengage and engage the first and second ring gears, respectively, in a synchronous manner, in order to shorten the gear-shifting period. However, the control of gear-shifting is beyond the scope of this study.

\subsection{Mathematical Model}

Assuming that the gears and their teeth are rigid, the constraint equations of the PGS are

$$
\begin{aligned}
& \dot{q}_{s} r_{s}+\dot{q}_{r} r_{r}=\dot{q}_{c}\left(r_{r}+r_{s}\right) \\
& \dot{q}_{s} r_{s}+\dot{q}_{p} r_{p}=\dot{q}_{c} r_{c}
\end{aligned}
$$

where $\dot{q}_{s}, \dot{q}_{r}, \dot{q}_{c}, \dot{q}_{p}$ are the angular velocities of the sun gear, the ring gear, the planet carrier, and the planet gear, respectively; $r_{s}, r_{r}, r_{c}, r_{p}$ are the radii of the sun gear, the ring gear, the planet carrier, and the planet gear, respectively.

The underdrive gear train is composed of two PGSs, thus involving eight components in total. Due to the sun shaft and planet-carrier physical connection, two additional constraints are present, namely,

$$
\begin{aligned}
& q_{s 1}=q_{s 2} \equiv q_{s} \\
& q_{c 1}=q_{c 2} \equiv q_{c}
\end{aligned}
$$

With constraints in Eqs. (12) and (13), the total set of the underdrive gear train is

$$
\begin{aligned}
& \dot{q}_{s} r_{s 1}+\dot{q}_{r 1} r_{r 1}=\dot{q}_{c}\left(r_{r 1}+r_{s 1}\right) \\
& \dot{q}_{s} r_{s 2}+\dot{q}_{r 2} r_{r 2}=\dot{q}_{c}\left(r_{r 2}+r_{s 2}\right) \\
& \dot{q}_{s} r_{s 1}+\dot{q}_{p 1} r_{p 1}=\dot{q}_{c} r_{c 1} \\
& \dot{q}_{s} r_{s 2}+\dot{q}_{p 2} r_{p 2}=\dot{q}_{c} r_{c 2}
\end{aligned}
$$

where subscripts 1 and 2 stand for the first and second PGSs, respectively. 
Table 2: Coefficient definition

\begin{tabular}{|c|c|}
\hline Coefficient & Definition \\
\hline$a$ & $r_{s 1} / r_{r 1}$ \\
\hline$b$ & $r_{s 2} / r_{r 2}$ \\
\hline$c$ & $\left(r_{s 1}+r_{r 1}\right) / r_{r 1}$ \\
\hline$d$ & $\left(r_{s 2}+r_{r 2}\right) / r_{r 2}$ \\
\hline$A$ & $I_{c}+n\left(m_{p 1}+m_{p 2}\right) r_{c}^{2}+I_{r 1} c^{2}++I_{r 2} d^{2}+n I_{p 1}\left(r_{c 1} / r_{p 1}\right)^{2}+n I_{p 2}\left(r_{c 2} / r_{p 2}\right)^{2}$ \\
\hline$B$ & $a c I_{r 1}+b d I_{r 2}+n I_{p 1}\left(r_{c 1} r_{s 1} / r_{p 1}^{2}\right)+n I_{p 2}\left(r_{c 2} r_{s 2} / r_{p 2}^{2}\right)$ \\
\hline$C$ & $I_{s 1}+I_{s 2}+I_{r 1} e^{2}++I_{r 2} b^{2}+n I_{p 1}\left(r_{s 1} / r_{p 1}\right)^{2}+n I_{p 2}\left(r_{s 2} / r_{p 2}\right)^{2}$ \\
\hline$D$ & $a(A-B) / c+C-B$ \\
\hline$E$ & $b(A-B) / d+C-B$ \\
\hline
\end{tabular}

With six constraints in Eqs. (14)-(16), the transmission, with eight components and corresponding generalized coordinates, has two degrees of freedom that change, depending on which gear ratio is engaged. The mathematical model of the transmission is derived by means of a Lagrangian formulation. The generalized coordinates are $\mathbf{q}=\left[\begin{array}{ll}q_{s} & q_{c}\end{array}\right]^{T}$. The mathematical model is derived as [36]

$$
\begin{aligned}
& A \ddot{q}_{c}+B \ddot{q}_{s}=\tau_{d}-c_{s} \dot{q}_{s}-a \tau_{r 1}-b \tau_{r 2} \\
& C \ddot{q}_{c}+B \ddot{q}_{s}=\tau_{l}-c_{c} \dot{q}_{c}+c \tau_{r 1}+d \tau_{r 2}
\end{aligned}
$$

where: $A, B, C, a, b, c$, and $d$ are constant coefficients defined in Table 2, $c_{s}$ is the damping coefficient of the sun shaft bearing, $c_{c}$ being that of the carrier bearing; $\tau_{d}, \tau_{l}, \tau_{r 1}, \tau_{r 2}$ are the torques of the electric motor, the load, the first ring clutch, and the second ring clutch, respectively.

Inverse dynamics was conducted to determine the static clutch torque required to maintain the ring gear at zero acceleration. The static clutch torques for ring gears 1 and 2 are

$$
\begin{aligned}
\tau_{r 1}^{s} & =D \ddot{q}_{s}-\tau_{l}-\tau_{d}+c_{c} \dot{q}_{c}+c_{s} \dot{q}_{s} \\
\tau_{r 2}^{s} & =E \ddot{q}_{s}-\tau_{l}-\tau_{d}+c_{c} \dot{q}_{c}+c_{s} \dot{q}_{s}
\end{aligned}
$$

where $D$ and $E$ are constant coefficients defined in Table 2 ,

\section{Experimental Work and Simulation}

The simulation of the topology-change model is divided into two phases: 1) to compute the velocity jump $\Delta \mathbf{w}$, and 2) to conduct a simulation with the velocity jump $\Delta \mathbf{w}$ taken into account. This section is devoted to phase 1, phase 2 being detailed in Section 5 . The flow chart of simulation phase 1 is included in Fig. 6 The procedure begins with obtaining the experimental gear-shifting by means of commercial transmissions or transmission testbeds. The former have better precision and accuracy than the latter because the former are built and designed to work in actual vehicles, whereas the latter are primarily designed for laboratory experimental work. Nevertheless, both transmissions can be utilized for the purpose of topology-change modeling. After experimental gear-shifting data are obtained, the transmission model is formulated and the model parameters are adjusted according to the transmission operating conditions. The adjustable parameters are the clutch torques and controller gains, the former referring to the clutch motor torques and the clutch configuration.

Subsequently, the model is simulated with the adjusted parameters and the results compared with the experimental gear-shifting of interest. If the simulation results are acceptable, the procedure can continue; otherwise, the procedure returns to the previous step, where the parameters need to be readjusted. Two possible criteria to evaluate the simulation results are the velocity jump and the gear-shifting period. However, it will be shown that the simulation results with the transmission dynamic model cannot match the experimental results in both criteria simultaneously because the model does not consider the velocity jumps during gear-shifting, hence the need of developing a topology-change model for transmissions. For this reason, 

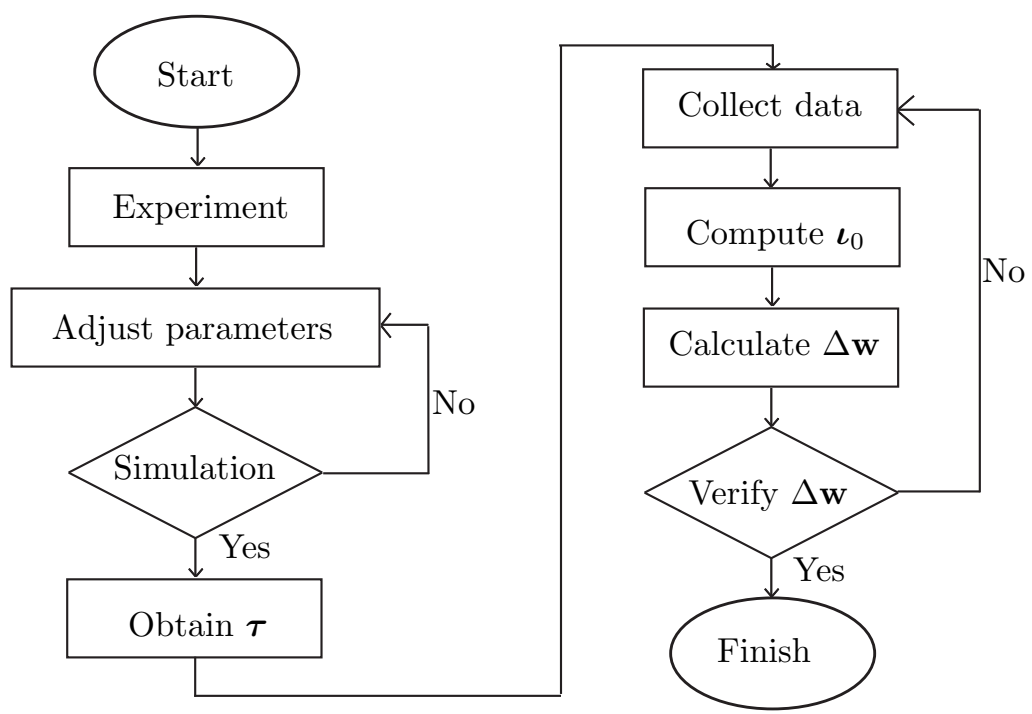

Figure 6: Flow chart of simulation phase 1

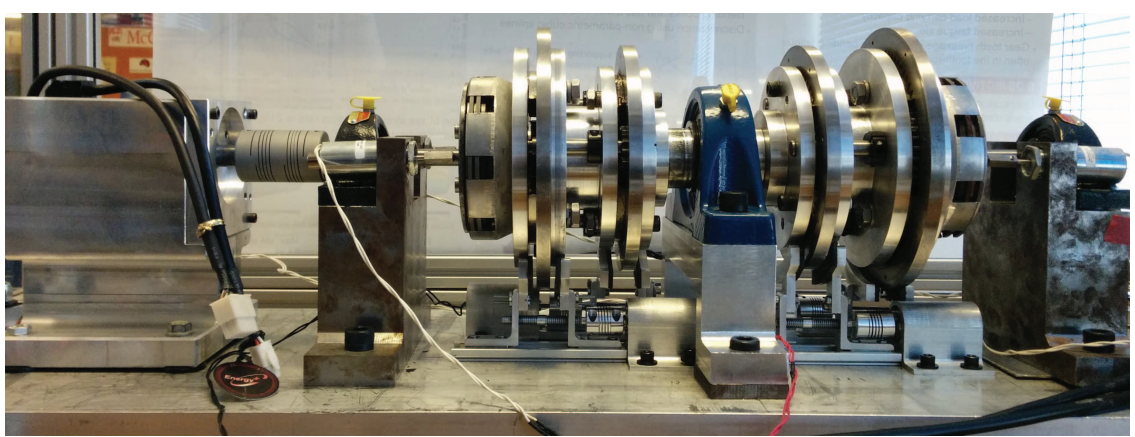

Figure 7: The testbed 23]

the simulation results are verified based on the gear-shifting period and the model is simulated to perform the gear-shifting over the gear-shifting period in the experimental test. The applied forces $\mathbf{f}$ and $\boldsymbol{\tau}$ can be computed in simulation for the next step.

Moreover, the next task is to collect a data set of values of $\boldsymbol{\tau}$ at discrete instants; these data are integrated to compute $\iota_{0}$. To this end, the interval of topology change, $t^{+}-t^{-}$, can be approximated by means of Eq. (11) by matching the velocity jump prediction with that in the experimental result. The process is iterative; therefore, an arbitrary and reasonable interval is chosen to start the loop. After $\boldsymbol{\iota}_{0}$ is computed, the velocity jump $\Delta \mathbf{w}$ is calculated by means of Eq. (11) and then verified experimentally. Once the results are acceptable, the simulation phase 1 is done. Otherwise, the process returns to the data-collection step.

\subsection{Velocity-jump Calculation}

In this study, an in-house developed testbed of the transmission, depicted in Fig. 7 , is utilized. The first sun gear transmits the input torque, whereas the carrier in the second set delivers the output torque, as

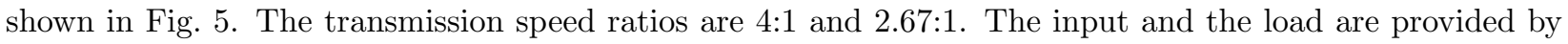
two identical Glentek brushless servomotors, GMBM80550-45. The input speed is maintained at $269 \mathrm{rpm}$ by means of a PID controller, while the load opposes the motion with a $2.26-\mathrm{Nm}$ torque. The input and output angular velocities are measured by the built-in motor encoders. Moreover, the ring clutch operates in a way similar to bicycle brakes, as shown in Fig. 8. The clamps are adjusted by means of a double-threaded rod and a DC motor, with screws of identical pitch and opposite hands. The opening and closing of the clutch is controlled by means of a DC motor. The brake pads are obtained from an off-the-shelf asphalt 


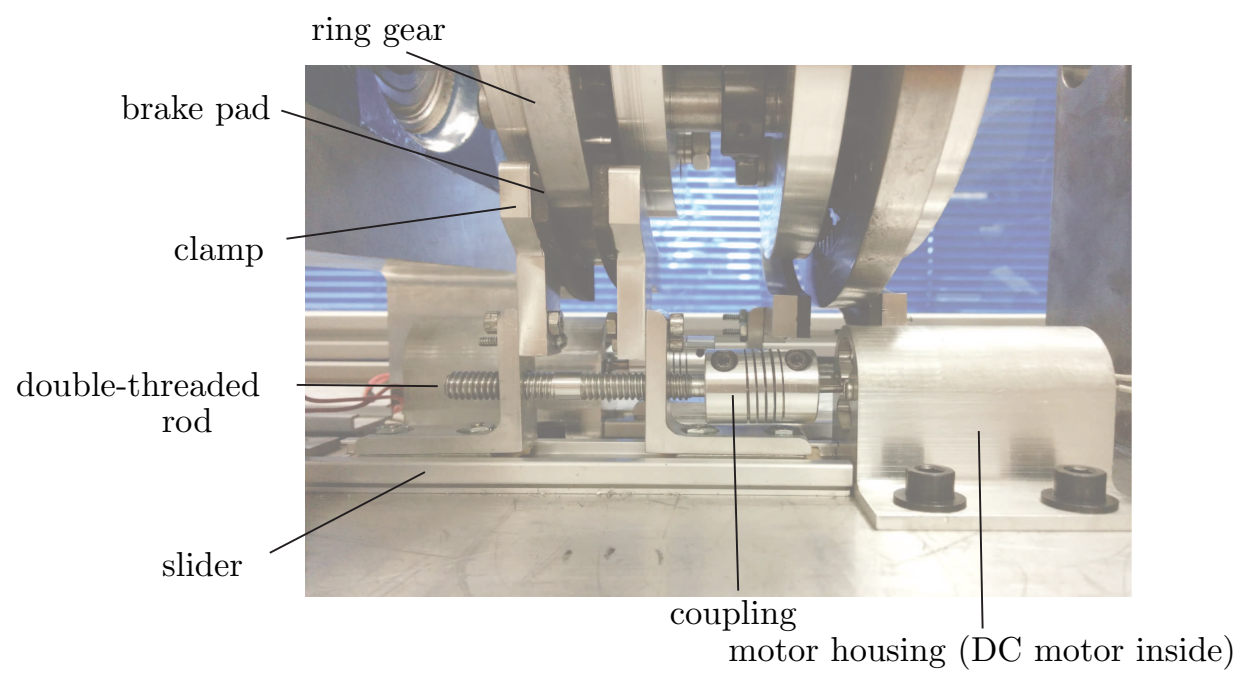

Figure 8: The ring clutch

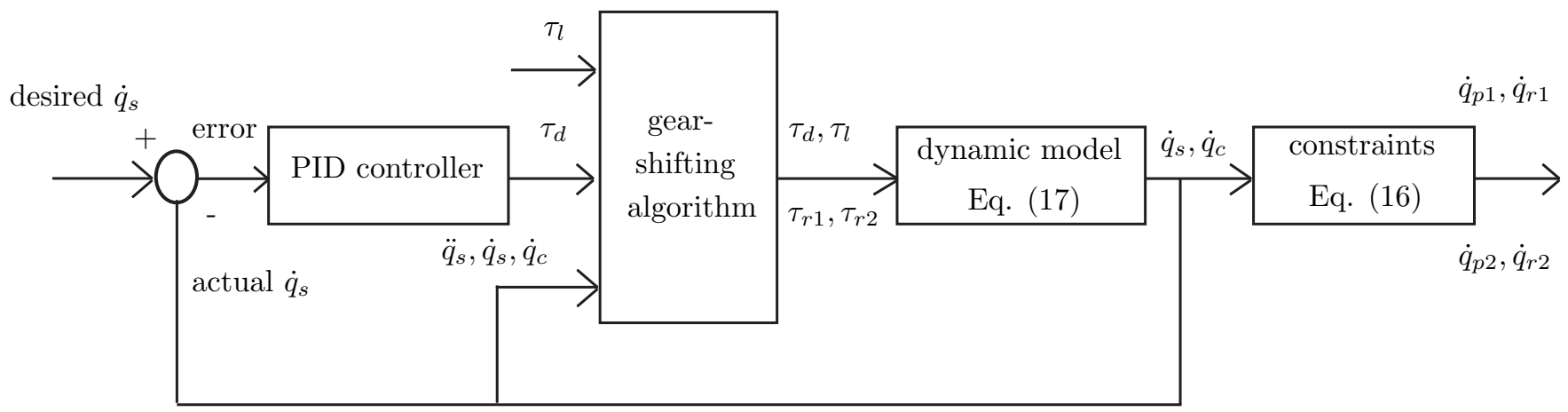

Figure 9: Control system block diagram in the simulation

Table 3: PID gains

\begin{tabular}{|c|c|}
\hline Parameter & Value \\
\hline Proportional & 0.1348 \\
\hline Integral & 16.5424 \\
\hline Derivative & -9.9659 \\
\hline
\end{tabular}

plate from McMaster Carr. Furthermore, the encoders and the ring clutch motors are connected to a Q8 data-acquisition board to receive and send signals. These are analyzed and processed in MATLAB/Simulink.

In addition, the simulation block diagram is illustrated in Fig. 9. A continuous-time PID controller is used to control the input torque $\tau_{d}$ that maintains the input speed $\dot{q}_{s}$ at the set value of $269 \mathrm{rpm}$. The tuned PID gains are listed in Table 3 The PID controller adjusts the input torque $\tau_{d}$ according to the error of the actual and desired values of $\dot{q}_{s}$. The input torque is then sent to the gear-shifting algorithm, which determines the ring clutch torque $1, \tau_{r 1}$, and ring clutch torque $2, \tau_{r 2}$. The gear-shifting algorithm for simulation phase 1 is provided in Table 4, the simulation conducted under MATLAB/Simulink, the algorithm written on the MATLAB Function block. The algorithm is quite simple, the gear-shifting is divided into two stages, namely, the pre- and post-event, which simulate the transmission before and after the topology change happens. In this study, the event is scheduled at $t=3.675 \mathrm{~s}$ (t_shift in the code). Prior to this, the first ring gear is engaged, whereas the second ring gear moves freely. The algorithm utilizes if logic as shown on code line 1. The pre-event stage is handled in code lines 1 to 7 , whereas the post-event stage in code lines 8 to 14 . Code lines 15 to 17 are used to complete the logic scheme.

In the pre-event stage, $\tau_{r 2}$ is zero, whereas $\tau_{r 1}$ grounds ring gear 1 . Ring gear 1 has two possible states, 
Table 4: Gear-shifting algorithm in simulation phase 1

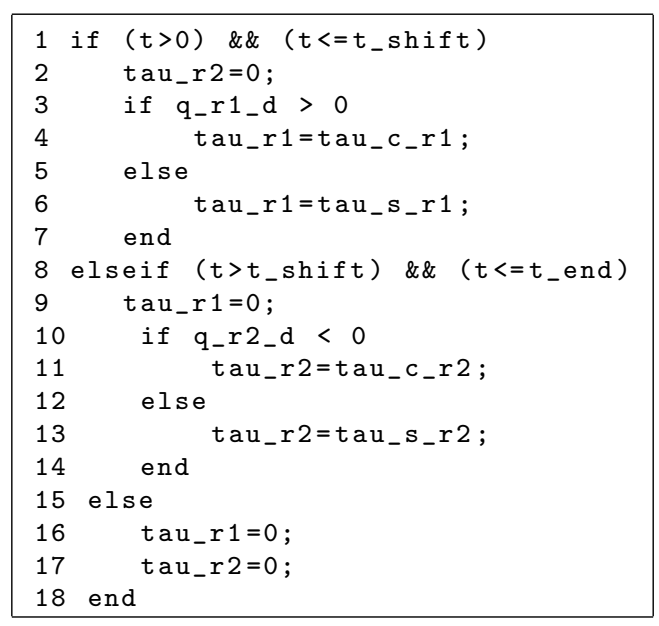

moving or stationary. Therefore, another if logic statement is introduced in line 3. Moreover, two kinds of clutch-torque information are needed, clutch torque capacity and static clutch torque. The former, denoted tau_c in the code (lines 4 and 11), is needed to oppose the motion of the gear and bring it to rest. Moreover, the static clutch torque, denoted tau_s in the code (lines 6 and 13), is needed to maintain the gear at zero acceleration. The values of the static clutch torque are computed by means of inverse dynamics, as per Eqs. (18) and (19). These two types of clutch torque are required to ground the ring gear. For instance, in the pre-event stage, $\tau_{r 1}$ will have the clutch torque capacity $\tau_{r 1}^{c}$ if ring gear 1 is moving, as given in code lines 3 and 4 . This torque opposes the motion of the ring gear such that the gear will slow down, eventually coming to rest. When at rest and about to move in the opposite direction due to the opposing torque of clutch torque capacity, $\tau_{r 1}$ is changed to the static clutch torque $\tau_{r 1}^{s}$ to lock down the gear, as shown in line 6. In this simulation test, the initial velocity is assumed to be zero; therefore, $\tau_{r 1}$ becomes the static clutch torque $\tau_{r 1}^{s}$ from the start of the simulation and locks the gear.

Likewise, the second stage of the gear-shifting algorithm follows an identical procedure. The ring clutch torques are now reversed, namely, $\tau_{r 1}$ is zero, whereas $\tau_{r 2}$ is adjusted. In this case, it is known that ring gear 2 is moving in the negative direction, therefore, $\tau_{r 2}$ has to oppose the ring gear. Torque $\tau_{r 2}$ is changed to $\tau_{r 2}^{s}$ when ring gear 2 reaches zero velocity to maintain the gear at zero acceleration. The gear-shifting algorithm needs the actual values of $\ddot{q}_{s}, \dot{q}_{s}$, and $\dot{q}_{c}$ in order to compute $\tau_{r 1}$ and $\tau_{r 2}$.

Furthermore, the algorithm will provide $\tau_{r 1}, \tau_{r 2}, \tau_{d}$ and $\tau_{l}$ to the transmission model, as shown in Fig 9 , The mathematical model of the novel modular MST is displayed in Eq. (17). The model parameters are obtained from the gear manufacturer and 3D CAD models. From this simulation, the dynamic response of the transmission is obtained. The values of $\dot{q}_{s}$ and $\dot{q}_{c}$ are submitted to the constraints in Eqs. (16) to compute the angular velocities of the planet and ring gears in the first and second PGSs. Moreover, the actual value of $\dot{q}_{s}$ is fed back to compute the error.

Furthermore, the constraint equation after gear-shifting is needed in Eq. (11) to compute the velocity jump $\Delta \mathbf{w}$. Therefore, the angular velocities of ring gears 1 and 2 are used in the constraint equation because one of the ring-gear angular velocities will be zero when the gear is shifted. Subsequently, the angular displacements of the sun, planet-carrier, ring gear 1 and ring gear 2 are chosen as the generalized coordinates, $\mathbf{q}=\left[\begin{array}{llll}q_{s} & q_{c} & q_{r 1} & q_{r 2}\end{array}\right]^{T}$. Matrices $\mathbf{M}$ and $\boldsymbol{\Phi}$ are given below, along with vector $\mathbf{f}$ :

$$
\mathbf{M}=\left[\begin{array}{cccc}
I_{s} & 0 & 0 & 0 \\
0 & I_{c} & 0 & 0 \\
0 & 0 & I_{r 1} & 0 \\
0 & 0 & 0 & I_{r 2}
\end{array}\right], \mathbf{\Phi}=\left[\begin{array}{cccc}
r_{s 1} & -\left(r_{s 1}+r_{r 1}\right) & r_{r 1} & 0 \\
r_{s 2} & -\left(r_{s 2}+r_{r 2}\right) & 0 & 0
\end{array}\right], \mathbf{f}=\left[\begin{array}{c}
\tau_{d} \\
-\tau_{l} \\
\tau_{r 1} \\
\tau_{r 2}
\end{array}\right]
$$

with $I_{s}, I_{r}, I_{c}$, and $I_{p}$ denoting the moments of inertia of the sun gear, the ring gear, the planet carrier, and the planet gear, respectively. 
Table 5: Simulation parameter values

\begin{tabular}{|c|c|c|c|c|c|c|}
\hline Parameter & Value & Units & & Parameter & Value & Units \\
\hline$r_{c}$ & 0.0508 & $\mathrm{~m}$ & & $I_{p 1}$ & $9.614 \times 10^{-5}$ & $\mathrm{~kg} \mathrm{~m}^{2}$ \\
\hline$r_{p 1}$ & 0.0239 & $\mathrm{~m}$ & $I_{p 2}$ & $5.864 \times 10^{-6}$ & $\mathrm{~kg} \mathrm{~m}^{2}$ \\
\hline$r_{p 2}$ & 0.0119 & $\mathrm{~m}$ & $I_{s 1}$ & $9.614 \times 10^{-5}$ & $\mathrm{~kg} \mathrm{~m}$ \\
\hline$r_{s 1}$ & 0.0239 & $\mathrm{~m}$ & $I_{s 2}$ & $4.927 \times 10^{-4}$ & $\mathrm{~kg} \mathrm{~m}$ \\
\hline$r_{s 2}$ & 0.0358 & $\mathrm{~m}$ & $I_{c}$ & 0.1262 & $\mathrm{~kg} \mathrm{~m}$ \\
\hline$r_{r 1}$ & 0.0716 & $\mathrm{~m}$ & $I_{r 1}$ & 0.0132 & $\mathrm{~kg} \mathrm{~m}^{2}$ \\
\hline$r_{r 2}$ & 0.0597 & $\mathrm{~m}$ & $I_{r 2}$ & 0.0082 & $\mathrm{~kg} \mathrm{~m}^{2}$ \\
\hline
\end{tabular}

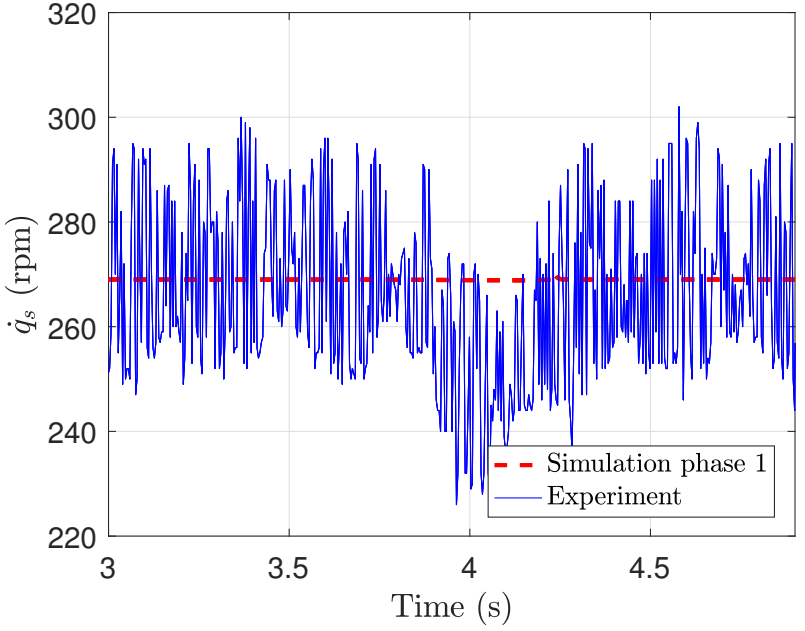

(a) Input speed

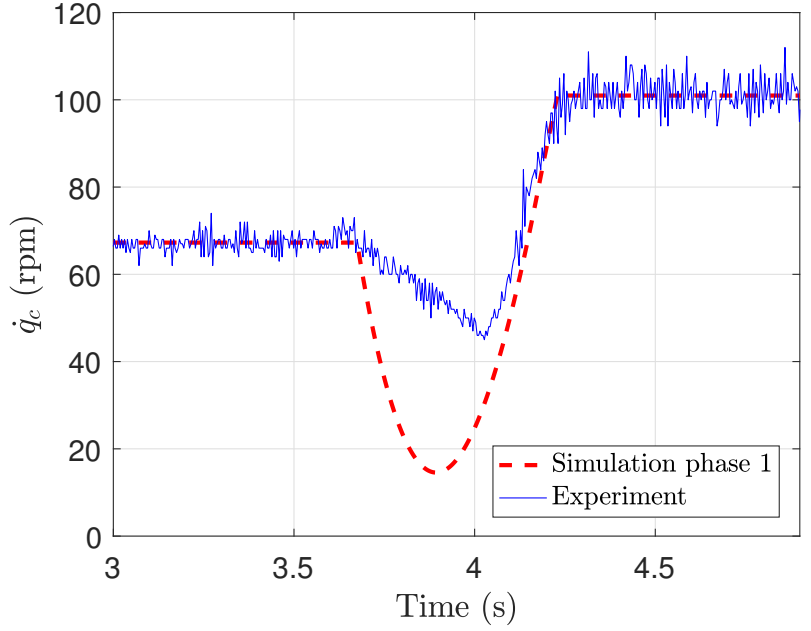

(b) Output speed

Figure 10: Simulation phase 1 results

Moreover, $\mathbf{T}$ and $\boldsymbol{\tau}$ are computed with the parameter values in Table 5 as follows:

$$
\mathbf{T}=\left[\begin{array}{cc}
6.02 & 0 \\
2.26 & 0 \\
1 & 0 \\
0 & 1
\end{array}\right], \boldsymbol{\tau}=\left[\begin{array}{c}
6.02 \tau_{d}-2.26 \tau_{l}+\tau_{r 1} \\
\tau_{r 2}
\end{array}\right]
$$

where $\mathbf{T}$ is obtained by means of the QR decomposition of $\boldsymbol{\Phi}^{T}[37]$; the lower $2 \times 2$ block of $\mathbf{T}$, multiplying a zero matrix block being freely chosen, as long as the resulting $\mathbf{T}$ is of full rank. Hence, the simplest choice is the $2 \times 2$ identity matrix.

The results of simulation phase 1 are shown in Fig. 10. The simulated input speed does not match well the experimental results, as the velocity jump does not occur in the simulation. In addition, the output speed drops to a significant amount as the gear-shifting period needs to match the experimental results, which makes it inaccurate for representing the actual transmission dynamic response. Hence, the topology-change model that takes the velocity jump into consideration is needed to predict reliably the response.

Figure 11(a) shows the clutch torque capacity. In this study, it is assumed that ring clutch 1 releases the ring gear immediately. Furthermore, the clutch torque capacity is adjustable to match the gear-shifting period of the experimental result. In addition, the speed ratio of the gear-shifting is shown in Fig. [11(b). This is obtained by dividing the input speed by the output speed. This figure shows the gear-shifting dynamics and the period where the topology of the transmission changes. Moreover, this verifies that the transmission is shifting into the new topology. 


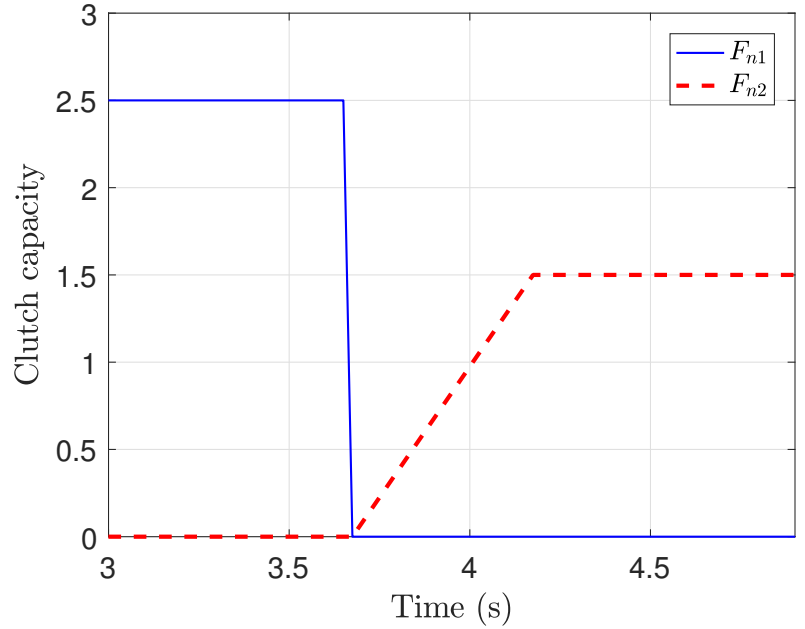

(a)

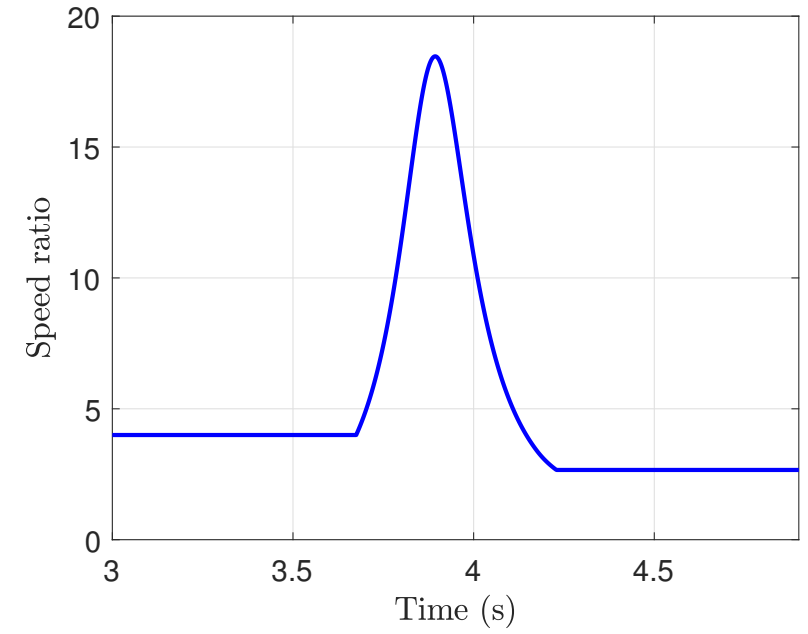

(b)

Figure 11: (a) Clutch torque capacities, (b) speed ratio

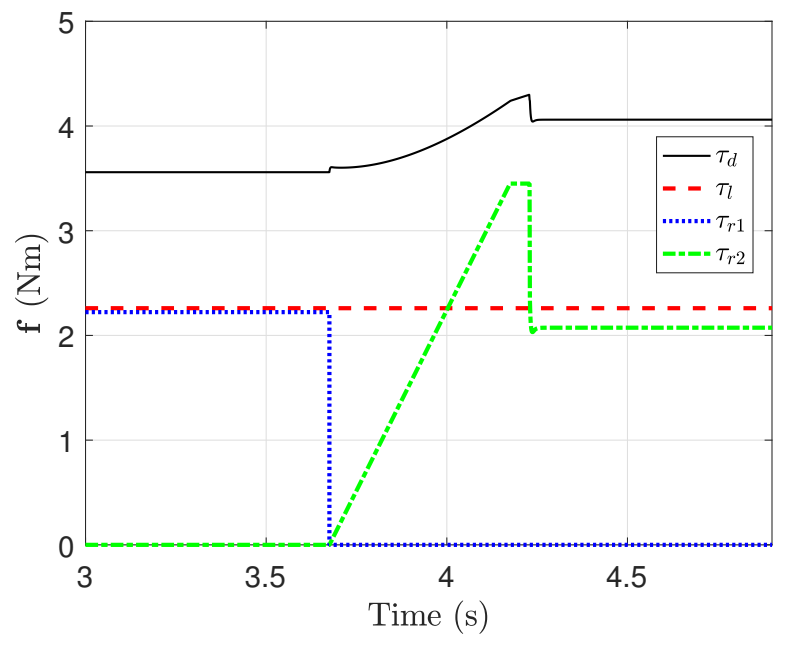

(a)

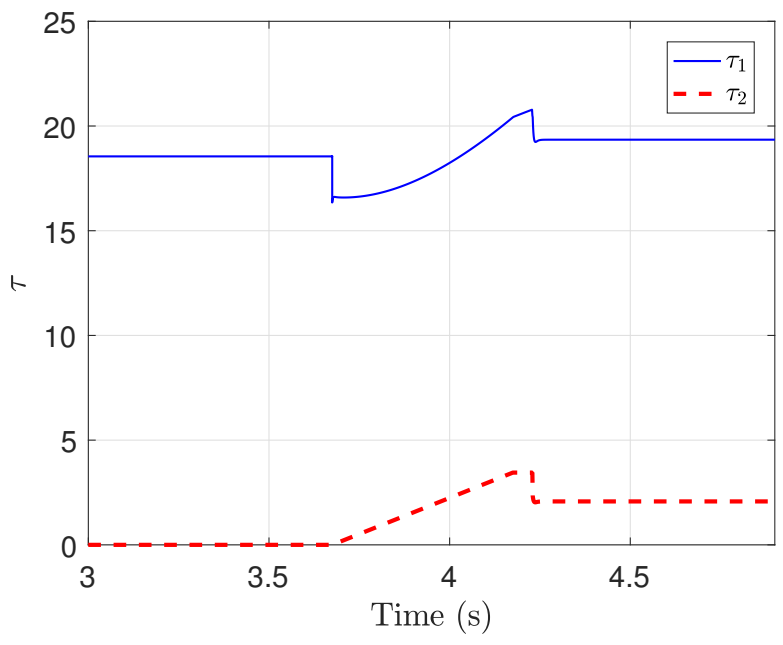

(b)

Figure 12: (a) The applied torques f , (b) $\boldsymbol{\tau}$

The applied force $\mathbf{f}$ is displayed in Fig. 12(a). As expressed in Eq. (20), $\mathbf{f}$ consists of four components, namely, $\tau_{d}, \tau_{l}, \tau_{r 1}$, and $\tau_{r 2}$. Torque $\tau_{d}$ is adjusted by the PID controller to maintain the input speed at a desired value, as shown in Fig. 9. Consequently, this torque was changing as shown in Fig. 12(a) to compensate the torque changes during gear-shifting. Moreover, the load torque $\tau_{l}$ in this study is kept constant throughout the simulation test. Furthermore, the ring clutch torques were adjusted based on the clutch torque capacity in Fig. 11(a) and on the static clutch torques in Eqs. (18) and (19). In the pre-event stage in Fig. 12(a), $\tau_{r 1}$ maintains the ring gear from moving, therefore, $\tau_{r 1}=\tau_{r 1}^{c}$. At the gear-shifting event, occurring at $t=3.675 \mathrm{~s}$, the post-event gear-shifting algorithm is employed, where ring clutch 1 releases the grips immediately and $\tau_{r 1}$ becomes zero. On the other hand, ring clutch 2 engages ring gear 2 and $\tau_{r 2}=\tau_{r 2}^{c}$ to oppose the motion of ring gear 2. After ring gear 2 comes to rest, $\tau_{r 2}$ becomes $\tau_{r 2}^{s}$ to maintain the zero acceleration. Both time histories of $\tau_{r 1}$ and $\tau_{r 2}$ can be observed in Fig. 12 (a).

Figure 12(b) gives the time history of $\tau$. Vector $\tau$ is expressed in Eq. (21) and integrated to find the velocity jump $\Delta \mathbf{w}$. In this study, the topology-change interval $\left(\Delta t=t^{+}-t^{-}\right)$is $0.0099 \mathrm{~s}$. The velocity jump 


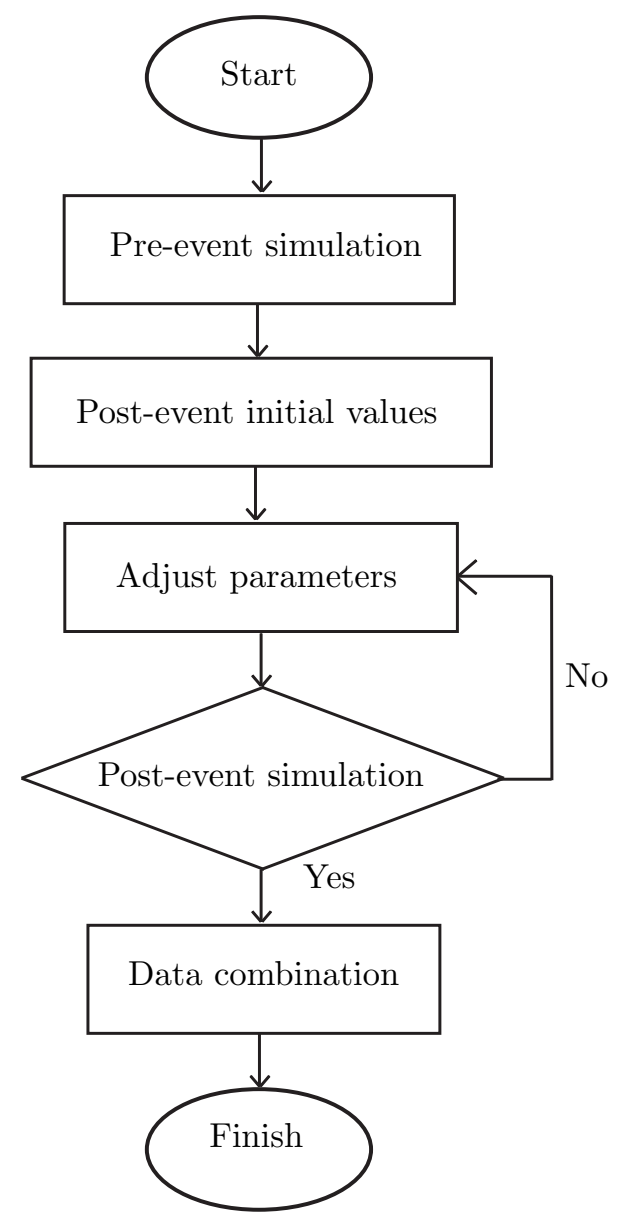

Figure 13: Flow chart of simulation phase 2

$\Delta \mathbf{w}$ in Eq. (11) can be readily obtained. The independent generalized velocities are $\mathbf{w}=\left[\dot{q}_{s} \dot{q}_{c}\right]^{T}$, while the velocity jump $\Delta \mathbf{w}$ is

$$
\Delta \mathbf{w}=\left[\begin{array}{c}
23.3313 \\
22.308
\end{array}\right] \mathrm{rpm}=\left[\begin{array}{c}
2.4432 \\
2.336
\end{array}\right] \mathrm{rad} / \mathrm{s}
$$

\section{Topology-change Model Implementation}

In this section we study simulation phase 2 , a simulation task that takes the velocity jump $\Delta \mathbf{w}$ computed in phase 1 into consideration. The flow chart of phase 2 is given in Fig. 13 Similar to simulation phase 1 , the test is divided into three stages, i.e., pre-event, event and post-event; the event stage simulates the transmission when the topology change happens.

The MATLAB/Simulink simulation scheme is similar to that in phase 1, except for the gear-shifting algorithm. The algorithms for the pre- and post-event simulation tasks are given in Tables 6 and 7 respectively. The gear-shifting algorithm for phase 2 is basically a division of the gear-shifting algorithm for phase 1 into two, namely, the pre-event simulation and the post-event simulation. This is needed to take into account the velocity jump obtained from phase 1 . In phase 2, two Simulink files are needed, one for pre-event, the other for post-event. The results from these simulation tasks are combined using a MATLAB m-file. Simulation phase 2 begins with the pre-event. The initial values are zero and the simulation is run until the gear-shifting time, which is $3.675 \mathrm{~s}$ in this study. Subsequently, the velocity jump $\Delta \mathbf{w}$ is added to the last value of $\mathbf{w}$ from the pre-event; this value becomes later the initial value of the post-event. The next step is parameter-adjustment to match the dynamic response in the experimental result. If the result in the post-event simulation does 
Table 6: Gear-shifting algorithm of pre-event simulation

\begin{tabular}{|c|c|}
\hline 1 & tau_r2=0; \\
\hline 2 & if $q_{-} r 1_{-} d>0$ \\
\hline 3 & $t_{a u} r 1=t a u_{-} c_{-} r 1 ;$ \\
\hline 4 & else \\
\hline 5 & tau_r1 $=t a u_{-} s_{-} r 1$; \\
\hline 6 & end \\
\hline
\end{tabular}

Table 7: Gear-shifting algorithm of post-event simulation

\begin{tabular}{|c|c|}
\hline 1 & t au_r $1=0$; \\
\hline 2 & if $q_{-} r 2_{-} d<0$ \\
\hline 3 & tau_r2=tau_c_r2; \\
\hline 4 & else \\
\hline 5 & tau_r2=tau_s_r2; \\
\hline 6 & end \\
\hline
\end{tabular}

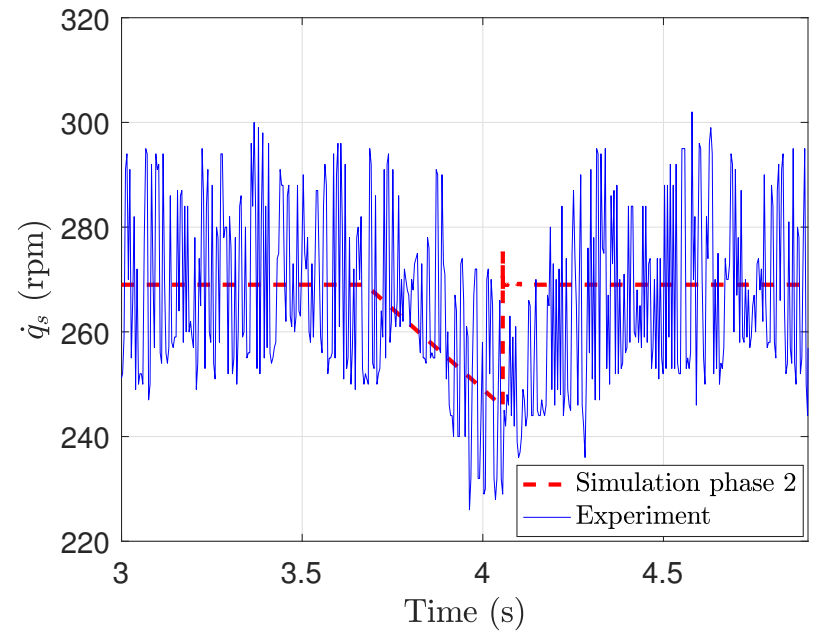

(a) Input speed

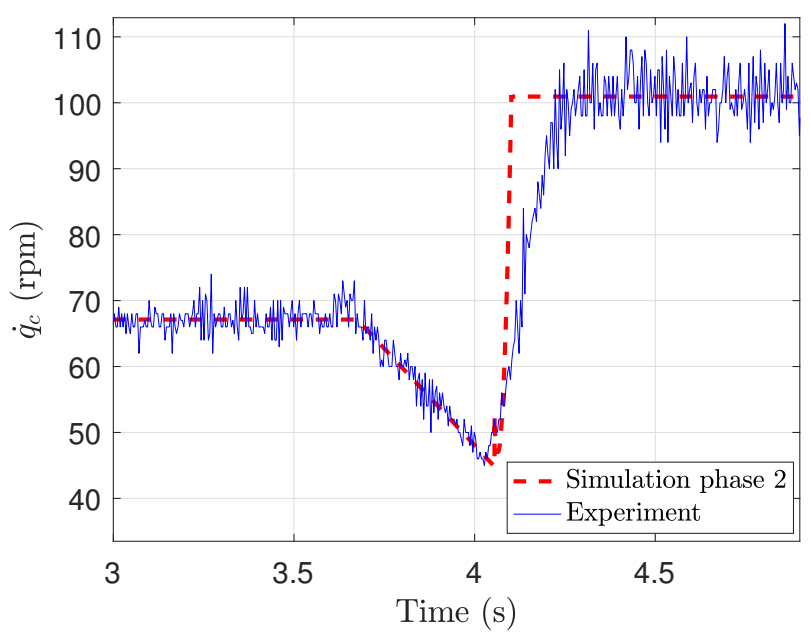

(b) Output speed

Figure 14: Simulation phase 2 results

not match the experimental result, the process will be returned to the variable adjustment stage until an acceptable result is obtained. Finally, the simulation results of pre- and post-events are combined to form the full simulation of the gear-shifting operation.

The full simulation results in this study are shown in Fig. 14. It can be observed that the dynamic responses of the model with the velocity-jump in Eq. (11) matches well the experimental results. These are more accurate predictions than those of the dynamic model in Eq. (17), as depicted in Fig. 10. The sun gear encountered a jump of $23.3313 \mathrm{rpm}=2.4432 \mathrm{rad} / \mathrm{s}$ during gear-shifting. The PID controller compensated the error and controlled the sun gear at the desired value within $0.1 \mathrm{~s}$. In contrast, the carrier underwent a velocity jump of $22.308 \mathrm{rpm}=2.336 \mathrm{rad} / \mathrm{s}$. In this study, the velocity jump is added $0.4 \mathrm{~s}$ after the gearshifting event, to account for the time elapsed for the velocity jump. This number is obtained from observing the experimental result.

The velocity jumps in the simulation results show the actual dynamic response in the transmission that cannot be captured by means of smooth mathematical models alone, such as that in Eq. (17). This information on velocity jumps is essential to increase the correctness of the dynamic response. Moreover, correct values of dependent generalized coordinates and their velocity jumps can also be predicted by means of the constraints in Eq. (16), as shown in Fig. 9. Plots of the angular velocities of the planet and ring gears in the first and second PGSs are depicted in Fig. 15. Having an accurate prediction of the dynamic response of the planet and ring gears in vehicle transmissions is essential for two reasons. First, they cannot be predicted because they 


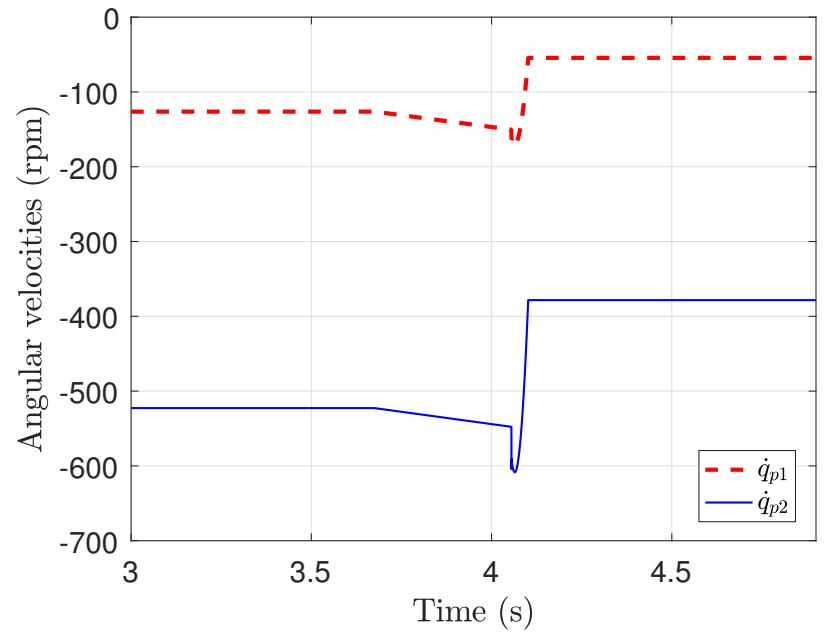

(a)

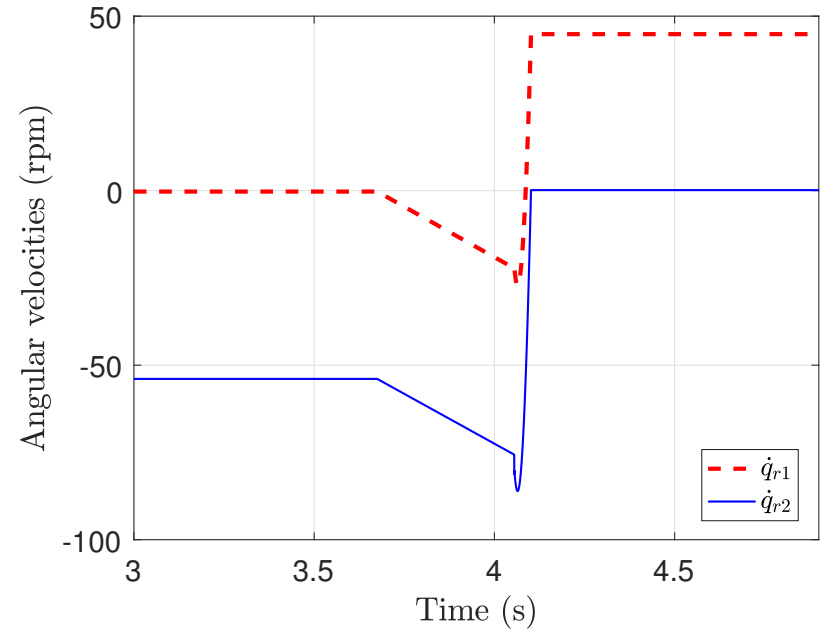

(b)

Figure 15: Angular velocities of (a) planet gears, (b) ring gears

cannot be measured. Second, having an accurate prediction of their response can provide a better control and system design for the transmission performance improvement. In commercial automatic transmissions, gear-shifting is controlled very precisely; therefore, the gear-shifting period and velocity jump are identical throughout the operation. Thus, results obtained in the simulation can be used to predict the dynamic response of the transmission in other conditions or operations. Furthermore, the topology-change model is applicable to any vehicle transmissions with gear-shifting. As shown in this study, the topology-change model needs three main terms from the transmission, namely, the inertia matrix, the orthogonal complement of the transmission constraint Jacobian, and the forces applied on the transmission. The first two terms can be determined from the transmission design data; the case study has provided a procedure to determine the last term.

\section{Conclusions}

Realistic mathematical models of multi-speed transmissions in electric vehicles are needed to understand the dynamic nature of the transmission system and to support their optimum design and control. However, an important phenomenon in transmissions has been overlooked, namely, the topology change during gearshifting. Therefore, a topology-change model based on the impulse-momentum relation is developed to address the non-smooth changes in multi-speed transmissions in electric vehicles during gear-shifting. A case study is included, whereby the topology-change model is implemented on an experimental modular multi-speed transmission for electric vehicles. In order to calculate the impulse, experimental and simulation tests are conducted by means of the transmission testbed and MATLAB/Simulink, respectively, where a gear-shifting is recorded experimentally and replicated by means of a mathematical model in simulation. Moreover, the velocity jump of the gear-shifting is computed by means of the model. Subsequently, simulation of the transmission gear-shifting incorporating the velocity jump is conducted. The topology-change model is essential to predict the actual motion of the transmission during the topology changes brought about by gear-shifting. Furthermore, the topology-change model developed in this paper is applicable to any vehicle transmissions with gear-shifting.

\section{Acknowledgments}

The design and research work reported here was supported by a grant under the Automotive Partnership Canada Project APCPJ418901-11. We gratefully acknowledge the support of our industrial partners: Linamar, TM4 and Infolytica. In addition, we acknowledge NSERC grant No. 4532-2010 and the McGill 
Engineering Doctoral Award granted to the first author. The last author acknowledges the support of a James McGill Professorship.

\section{References}

[1] R. Schallenberg, Prospects for the electric vehicle: a historical perspective, IEEE Transaction on Education 23 (3) (1980) 137-143.

[2] B. Skugor, J. Deur, A novel model of electric vehicle fleet aggregate battery for energy planning studies, Energy 92 (3) (2015) 444-455.

[3] J. Larminie, J. Lowry, Electric Vehicle Technology Explained, Second Edition, John Wiley and Sons, West Sussex, England, 2012.

[4] I. Husain, Electric and Hybrid Vehicles: Design Fundamentals, Second Edition, Taylor and Francis, 2011.

[5] M. Roozegar, J. Angeles, A two-phase control algorithm for gear-shifting in a novel multi-speed transmission for electric vehicles, Mechanical Systems and Signal Processing 104 (2018) 145-154.

[6] B. Akin, S. Ozturk, H. Toliyat, M. Rayner, DSP-based sensorless electric motor fault-diagnosis tools for electric and hybrid electric vehicle powertrain applications, IEEE Transactions on Vehicular Technology 58 (5) (2009) 2150-2159.

[7] D. Kim, S. Hwang, H. Kim, Vehicle stability enhancement of four-wheel-drive hybrid electric vehicle using rear motor control, IEEE Transactions on Vehicular Technology 57 (2) (2008) 727-735.

[8] M. S. R. Mousavi, H. V. Alizadeh, B. Boulet, Estimation of synchromesh frictional torque and output torque in a clutchless automated manual transmission of a parallel hybrid electric vehicle, IEEE Transactions on Vehicular Technology 66 (7) (2017) 5531-5539.

[9] H. Yoo, S. Sul, Y. Park, J. Jeong, System integration and power-flow management for a series hybrid electric vehicle using supercapacitors and batteries, IEEE Transactions on Industry Application 44 (1) (2008) 108-114.

[10] S. Jung, H. Lee, C. Song, J. Han, W. Han, G. Jang, Optimal operation plan of the online electric vehicle system through establishment of a DC distribution system, IEEE Transaction of Power Electronics 28 (12) (2013) 5878-5889.

[11] M. Roozegar, Y. D. Setiawan, J. Angeles, Design, modelling and estimation of a novel modular multispeed transmission system for electric vehicles, Mechatronics 45 (2017) 119-129.

[12] A. Sorniotti, T. Holdstock, G. Pilone, F. Viotto, S. Bertolotto, M. Everitt, R. Barnes, B. Stubbs, M. Westby, Analysis and simulation of the gearshift methodology for a novel two-speed transmission system for electric powertrains with a central motor, Proceedings of the Institution of Mechanical Engineers, Part D: Journal of Automobile Engineering 226 (7) (2012) 915-929.

[13] M. Mousavi, A. Pakniyat, T. Wang, B. Boulet, Seamless dual brake transmission for electric vehicles: design, control and experiment, Mechanism and Machine Theory 94 (2015) 96-118.

[14] S. Roberts, Multispeed transmission for electric vehicles, ATZ worldwide 114 (4) (2012) 8-11.

[15] C.-Y. Tseng, C.-H. Yu, Advanced shifting control of synchronizer mechanisms for clutchless automatic manual transmissions in an electric vehicle, Mechanism and Machine Theory 84 (2015) 37-56.

[16] Z. Lei, D. Sun, Y. Liu, D. Qin, Y. Zhang, Y. Yang, L. Chen, Analysis and coordinated control of mode transition and shifting for a full hybrid electric vehicle based on dual clutch transmissions, Mechanism and Machine Theory 114 (2017) 125-140. 
[17] B. Zhu, Research of two speed DCT electric power-train and control system, PhD Thesis, University of Technology, Sydney (2015).

[18] B. Stubbs, P. M. F. Ceng, eDCT: 4 speed seamless-shift technology for electric vehicles, Hybrid and Electric Vehicles Conference (HEVC), 6-7 Nov., Stevenage, UK (2013) 1-5.

[19] M. Inalpolat, A. Kahraman, Dynamic modelling of planetary gears of automatic transmissions, Proceedings of the Institution of Mechanical Engineers, Part K: Journal of Multi-body Dynamics 222 (3) (2008) 229-242.

[20] M. Bachinger, M. Stolz, M. Horn, A novel drivetrain modelling approach for real-time simulation, Mechatronics 32 (2015) 67-78.

[21] G. Lucente, M. Montanari, , C. Rossi, Modelling of an automated manual transmission system, Mechatronics 17 (2-3) (2007) 73-91.

[22] J. J. Oh, S. B. Choi, J. Kim, Driveline modeling and estimation of individual clutch torque during gear shifts for dual clutch transmission, Mechatronics 24 (5) (2014) 449-463.

[23] Y. D. Setiawan, M. Roozegar, T. Zou, J. Angeles, A mathematical model of multi-speed transmissions in electric vehicles in the presence of gear-shifting, IEEE Transactions on Vehicular Technology, in press (2017).

[24] K. Zhao, H. Wang, G. Chen, Dynamics simulation of topology-varying mechanisms by replacing local modeling units, International Design Engineering Technical Conferences and Computers and Information in Engineering Conference (2008) 511-519.

[25] Y. Zhang, W. F. Xu, Z. Y. Wang, Y. She, Dynamic modeling of self-reconfigurable multi-arm space robotic system with variable topology, IEEE International Conference on Robotics and Biomimetics (2013) 599-604.

[26] W. Guo, T. Wang, A methodology for simulations of multi-rigid body systems with topology changes, Multibody System Dynamics 35 (1) (2015) 25-38.

[27] J. M. Font-Llagunes, J. Kovecses, Dynamics of non-ideal topology transitions in multibody mechanical systems, International Design Engineering Technical Conferences and Computers and Information in Engineering Conference (2009) 1-8.

[28] J. Kovecses, J. M. Font-Llagunes, An eigenvalue problem for the analysis of variable topology mechanical systems, Journal of Computational and Nonlinear Dynamics 4 (3) (2009) 1-9.

[29] R. Mukherjee, K. Anderson, Efficient methodology for multibody simulations with discontinuous changes in system definition, Multibody System Dynamics 18 (2007) 145-168.

[30] J. Shi, J. Hong, Z. Liu, Multi-variable approach of contact-impact issue in variable topology system, Theoretical and Applied Mechanics Letters 3 (1) (2013) 58-62.

[31] F. Qi, T. Wang, J. Li, The elastic contact influences on passive walking gaits, Robotica 29 (5) (2011) $787-796$.

[32] R. M. Mukherjee, K. S. Anderson, Efficient methodology for multibody simulations with discontinuous changes in system definition, International Conference on Multibody Systems, Nonlinear Dynamics and Control (2008) 129-139.

[33] H. Wang, P. Eberhard, Z. Lin, Modeling and simulation of closed loop multibody systems with bodies joints composite modules, Multibody System Dynamics 24 (2010) 389-411.

[34] F. M. L. Amirouche, Fundamentals of Multibody Dynamics: theory and applications, Birkhuser, Boston, 2006. 
[35] A. Morozov, T. Zou, M. Mousavi, Y. D. Setiawan, J. Angeles, B. Boulet, Design and gear shift control of a modular swift-shift multi-speed transmission for EVs, European Battery, Hybrid and Fuel Cell Electric Vehicle Congress, Brussels, Belgium, December 1-4 (2015) 1-10.

[36] M. Roozegar, J. Angeles, The optimal gear-shifting for a multi-speed transmission system for electric vehicles, Mechanism and Machine Theory 116 (2017) 1-13.

[37] G. H. Golub, C. F. Van Loan, Matrix Computations, Johns Hopkins Univ Press, Baltimore, 2013.
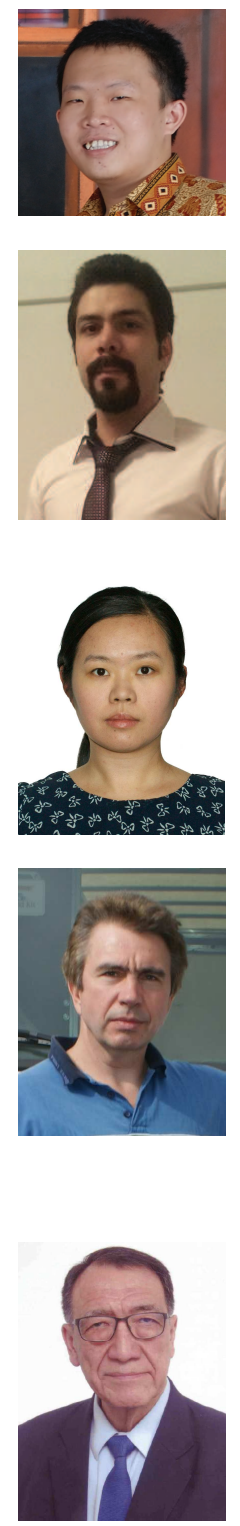

Yuhanes D. S. Liauw received the B.Eng. degree in mechanical engineering from Diponegoro University, Semarang, Indonesia, in 2012 and the M.Eng. degree in mechanical design engineering from Pukyong National University, Busan, South Korea, in 2015. He is currently working toward the Ph.D. degree in mechanical engineering with McGill University, Montreal, Canada. His research focuses on developing a mathematical model of multi-speed transmissions in electric vehicles in the presence of gear-shifting.

Mehdi Roozegar has received his B.Sc. and M.Sc. degrees in Mechanical Engineering from Shiraz University, Shiraz, Iran, in 2011 and University of Tehran, Tehran, Iran, in 2013, respectively. He is presently working towards his Ph.D. degree in the Centre for Intelligent Machines (CIM) at McGill University, Montreal, Canada. His research interests include modelling, estimation and control of multi-speed transmissions (MSTs) in electric vehicles $(\mathrm{EVs})$, robotics, mechatronics, optimization and machine learning.

Ting Zou, Ph.D., is a Postdoctoral Fellow working with Prof. Angeles at the Centre for Intelligent Machines, McGill University. Dr. Zou obtained a B.Sc. in electrical engineering from Xi'an Jiaotong University in 2005, a M.Sc. in automatic control engineering from Xi'an Jiaotong University in 2008, and a PhD degree in mechanical engineering from McGill University in 2013. Her research interests include design and optimization of robotic systems, automotive transmission system design and optimization.

Alexei Morozov, Eng., Ph.D., is Research Associate at McGill University, and a Lecturer in the Department of Mechanical Engineering. Dr. Morozov graduated from Bauman Technical University, Moscow, in 1983, in computer science, and received a Ph.D. degree from the Graduate School of Mechanical Engineering Research Institute of Russian Academy of Sciences in Moscow in 1990, in robotics. Dr. Morozov is a registered Professional Engineer in the province of Quebec, his research areas including robotics, design and optimization of drivetrains for electric vehicles, load case analysis and X-by-wire technology.

Jorge Angeles received his Engineering Diploma in Electromechanical Engineering and the M.Eng. degree in Mechanical Engineering from Universidad Nacional Autonoma de Mexico (UNAM), Mexico City, in 1969 and 1970, respectively, the Ph.D. in Applied Mechanics from Stanford University in 1973. In 1984, he joined the Department of Mechanical Engineering, McGill University, in Montreal after 11 years of teaching at UNAM. In 1985, Angeles became a founding member of the Centre for Intelligent Machines at McGill. Since September 2017 he is Professor Emeritus of Mechanical Engineering. Dr. Angeles is one of the 12 honorary members of IFToMM and Fellow of: The Royal Society of Canada; the Canadian Society for Mechanical Engineers; ASME; IEEE; and the Canadian Academy of Engineering. 